JOURNAL OF ALGEBRAIC STATISTICS

Issue in honor of S. E. Fienberg

Volume 10, No. 1, 2019, p.115-127

https://jalgstat.library.iit.edu/

ISSN $\quad 1309-3452$

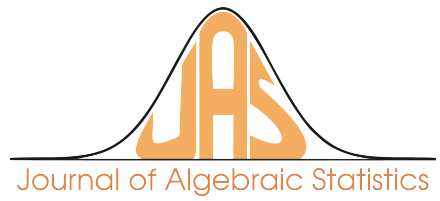

\title{
Cubature Rules and Expected Value of Some Complex Functions
}

\author{
Claudia Fassino $^{1}$, Eva Riccomagno ${ }^{1, *}$, Maria Piera Rogantin ${ }^{1}$ \\ 1 Department of Mathematics, University of Genova, Italy
}

\begin{abstract}
The expected value of some complex valued random vectors is computed by means of the indicator function of a designed experiment as known in algebraic statistics. The general theory is set-up and results are obtained for finite discrete random vectors and the Gaussian random vector. The precision space of some cubature rules/designed experiments is determined.
\end{abstract}

2000 Mathematics Subject Classifications: 13P10, 41A05, 65D32

Key Words and Phrases: Design of experiments, Indicator function, Interpolatory cubature formulæ, Precision space, Complex functions, Evaluation of expected values

\section{Introduction}

Evaluations of integrals is a recurrent task in statistics and probability for example when computing marginal distributions, in the analysis of contingency tables, when estimating the moments of some known distribution or when evaluating the marginal likelihood integrals in Bayesian inference, spectral analysis of time series [1] and probability. More than in statistics, complex valued random vectors and their integration find application in many other fields such as electromagnetism and quantum mechanics, and largely in digital communication [11] and signal processing (e.g. [17] and for a setting similar to ours [16]). Interestingly the usefulness of complex random vectors has also been argued in actuarial science [9] besides time series analysis. An introduction to the statistical analysis based on complex Gaussian distributions is given in [8] and a recent paper on second order estimation with complex-valued data focused on digital signal processing can be found in $[10]$.

In this paper we address the problem of computing the expected value with respect to a generic probability measure $\lambda$, of a function $g$ defined on $G^{k}$, for suitable space $G$. The measure $\lambda$ could be discrete or continuous. Specific results are given for the multivariate complex Gaussian distributions.

\footnotetext{
${ }^{*}$ Corresponding author.
}

Email addresses: fassino@dima.unige.it (C. Fassino), riccomagno@dima.unige.it (E. Riccomagno), rogantin@dima.unige.it (M. P. Rogantin) 
Claudia Fassino, Eva Riccomagno, Maria Piera Rogantin / J. Alg. Stat., 10, No.1 (2019), pp.115-127 115

The expected value of $g$ is approximated by an interpolatory cubature rule of the form

$$
\int_{G^{k}} g d \lambda=\sum_{d \in \mathcal{D}} w_{d} g(d)+R(g)
$$

where $\mathcal{D}$ is a finite set giving the cubature nodes, the $\left\{w_{d}\right\}_{d \in \mathcal{D}}$ are called weights and $R(g)$ is the error committed when approximating the integral with the finite sum.

The algebraic statistics theory of design of experiments allows, when $G=\mathbb{C}$, the exploitation of the so-called fractional factorial designs as sets of nodes. In such a framework a suitable choice for the coordinates of the nodes is the $m$-th roots of the unity, $m \in \mathbb{Z}_{\geq 0}$, see [14].

The weights $\left\{w_{d}\right\}_{d \in \mathcal{D}}$ are obtained from a vectorial basis of interpolator polynomials. A special basis is the basis of the quotient space $\mathbb{C}\left[z_{1}, \ldots, z_{k}\right] / I(\mathcal{D})$, where $I(\mathcal{D})$ is the polynomial ideal of $\mathcal{D}$, see [13].

Given a set of nodes and weights, it is of interest to determine classes of functions $g$ for which the error is zero. This set is called the precision space of the cubature rule. Quadrature rules (i.e. bi-dimensional cubature rules) with complex valued nodes have been studied e.g. in [12]. Here we work in a multi-dimensional setting.

Our work unveils a connection between cubature rules and design of experiments which, to our knowledge, has been unnoticed so far in the literature. We find this connection somewhat natural because both in cubature rule theory and design of experiment theory a key point is to determine a suitable finite set of points $\mathcal{D}$ and their weights $\left\{w_{d}\right\}_{d \in \mathcal{D}}$ for achieving some specific task, although this can be different between the two theories and also within them. Another common task to the two theories is, given $\mathcal{D}$ and $\left\{w_{d}\right\}_{d \in \mathcal{D}}$, find their range of applicability, e.g. power of estimation, precision space.

This paper deals with this second task and it does so by the synergic use of tools and techniques from commutative algebra, numerical analysis and algebraic statistics. In particular, some results in $[3,5]$ are generalised to the complex case. The link between the above cubature problem and the algebraic statistics theory of fractional factorial design of experiments is made through the representation of a fractional factorial experiment as a polynomial indicator function [14]. This is similar to [6] which instead unearthed the connection between Markov bases for contingency tables and design of experiments.

In Section 4.1 we focus our attention on the special case with equal weights and we obtain some specific results for the Gaussian density in Section 4.2. While in Section 2 we provide necessary and sufficient conditions for obtaining such cubature rules and we analyse their precision space, that is the vector space of polynomials $p$ whose expected value is equal to $\sum_{d \in \mathcal{D}} w_{d} f p(d)$, namely with zero error $R(p)$. The weights are found in Section 3 .

\section{Interpolatory rules}

Let $\lambda$ be a measure on $\mathbb{C}^{k}$ with finite moments (at least up to a certain degree) and $g$ be a complex integrable function, $g: \mathbb{C}^{k} \rightarrow \mathbb{C}$. Let $\mathcal{D} \subset \mathbb{C}^{k}$ be a set with $n$ elements and let $w \in \mathbb{C}^{n}$ be the vector $\left[w_{d}\right]_{d \in \mathcal{D}}$. 
Claudia Fassino, Eva Riccomagno, Maria Piera Rogantin / J. Alg. Stat., 10, No.1 (2019), pp.115-127 116

A cubature rule $(\mathcal{D}, w)$ is a formula of the type

$$
\int_{\mathbb{C}^{k}} g d \lambda=\sum_{d \in \mathcal{D}} w_{d} g(d)+R_{\mathcal{D}, w}(g)
$$

where the sum provides an approximation to the integral and $R_{\mathcal{D}, w}$ is the respective error. The $w_{d}$ 's are called the weights and the elements of $\mathcal{D}$ the nodes of the cubature rule.

Let $\mathbb{C}\left[z_{1}, \ldots, z_{k}\right]$ be the ring of polynomials with complex coefficients in the indeterminates $z_{1}, \ldots, z_{k}$ and let $\mathcal{P}$ be a set of polynomials contained in $\mathbb{C}\left[z_{1}, \ldots, z_{k}\right]$. A cubature rule $(\mathcal{D}, w)$ is exact for $\mathcal{P}$ if for all elements $p$ of $\mathcal{P}$

$$
\int_{\mathbb{C}^{k}} p d \lambda=\sum_{d \in \mathcal{D}} w_{d} p(d)
$$

or, equivalently, if $R_{\mathcal{D}, w}(p)=0$.

A cubature rules $(\mathcal{D}, w)$ is called interpolatory if it is exact for a set $\mathcal{P}$ of interpolatory polynomials over $\mathcal{D}$. This definition generalises the definition of univariate interpolatory quadrature rules, where $\mathcal{P}$ is the set of univariate polynomials with degree strictly lower than the cardinality of $\mathcal{D}$, that is the set of the interpolatory polynomials over $\mathcal{D}[7]$.

In this paper, given a set of nodes $\mathcal{D}$, we only consider sets of polynomials $\mathcal{P}$ such that, for any function $g: \mathbb{C}^{k} \rightarrow \mathbb{C}$, there exists a unique interpolatory polynomial $p_{g, \mathcal{D}} \in \mathcal{P}$ with $g(d)=p_{g, \mathcal{D}}(d)$ for all $d \in \mathcal{D}$. The pair $(\mathcal{D}, \mathcal{P})$ is called correct. For instance, the pair $(\mathcal{D}, \mathcal{P})$ is correct if $\mathcal{D}=\left\{d_{1}, \ldots, d_{n}\right\} \subset \mathbb{C}$ and $\mathcal{P}=\operatorname{Span}_{\mathbb{C}}\left(x^{\alpha} \mid \alpha=0, \ldots, n-1\right)$.

Let $\alpha \in \mathbb{Z}_{\geq 0}^{k}$, let $z^{\alpha}=z_{1}^{\alpha_{1}} \ldots z_{k}^{\alpha_{k}}$ be a monomial in the indeterminates $z_{1}, \ldots, z_{k}$ and let $\mathbb{T}=\left\{z^{\alpha} \mid \alpha \in \mathbb{Z}_{\geq 0}^{k}\right\}$ be the set of all monomials. Let $S \subset \mathbb{T}$ be a set of monomials such that each $p \in \mathcal{P}$ can be expressed as $p=\sum_{s \in S} c_{s} s, c_{s} \in \mathbb{C}$, that is $\mathcal{P}$ is a vectorial space over $\mathbb{C}$ with basis $S$. We denote $\mathcal{P}=\operatorname{Span}_{\mathbb{C}}(S)$.

An interpolatory polynomial $p_{g} \in \operatorname{Span}_{\mathbb{C}}(S)$ of a function $g$ over $\mathcal{D}$ is such that $p_{g}(d)=$ $g(d)$, that is $\sum_{s \in S} c_{s} s(d)=g(d)$, for each $d \in \mathcal{D}$. Denoting by $X_{\mathcal{D}, S}=[s(d)]_{d \in \mathcal{D}, s \in S}$ the evaluation matrix of the elements of $S$ at $\mathcal{D}$ and by $[g(d)]_{d \in \mathcal{D}}$ the evaluation vector of $g$ at $\mathcal{D}$, the coefficient vector $c=\left[c_{s}\right]_{s \in S}$ of the polynomial $p_{g}$ satisfies the linear system $X_{\mathcal{D}, S} c=[g(d)]_{d \in \mathcal{D}}$. If the pair $(\mathcal{D}, \mathcal{P})$ is correct, for each function $g$, that is for each vector $[g(d)]_{d \in \mathcal{D}}$, there exists a unique coefficient vector $c$ solution of this linear system, and so the pair is correct if and only if $X_{\mathcal{D}, S}$ is a square non singular matrix, that is if and only if $\# S=\# \mathcal{D}$ and the evaluation vectors $[s(d)]_{d \in \mathcal{D}}$ are linear independent vectors.

In the following we consider cubature rules $(\mathcal{D}, w)$ which are interpolatory with respect to a polynomial set $\mathcal{P}$ such that the pair $(\mathcal{D}, \mathcal{P})$ is correct. A correct pair $(\mathcal{D}, \mathcal{P})$ can be obtained considering $\mathbb{C}$-vector spaces $\mathcal{P}$ which are isomorphic to the quotient space $\mathbb{C}\left[z_{1}, \ldots, z_{k}\right] / I(\mathcal{D})$, that is considering monomial sets $S$ isomorphic to a basis of the quotient space $\mathbb{C}\left[z_{1}, \ldots, z_{k}\right] / I(\mathcal{D})$. There exist algebraic algorithms to compute monomial bases of such a vector space, for instance the Buchberger-Möller algorithm [2]. 
Claudia Fassino, Eva Riccomagno, Maria Piera Rogantin / J. Alg. Stat., 10, No.1 (2019), pp.115-127 117

By definition, an interpolatory cubature rule $(\mathcal{D}, w)$ is exact for each polynomial in $\mathcal{P}$ but, in general, there exist polynomials $p \notin \mathcal{P}$ such that $R_{\mathcal{D}, w}(p)=0$. In order to study the set of these polynomials, we introduce the notions of precision basis and precision space.

A finite monomial set $\mathcal{B}_{\mathcal{D}, w} \subset \mathbb{T}$ is a precision basis for $(\mathcal{D}, w)$ if

$\int_{\mathbb{C}^{k}} z^{\alpha} d \lambda=\sum_{d \in \mathcal{D}} w_{d} z^{\alpha}(d)$ for all $z^{\alpha} \in \mathcal{B}_{\mathcal{D}, w}$ and $\int_{\mathbb{C}^{k}} z^{\alpha} d \lambda \neq \sum_{d \in \mathcal{D}} w_{d} z^{\alpha}(d)$ for all $z^{\alpha} \notin \mathcal{B}_{\mathcal{D}, w}$.

The precision basis is the largest set of monomials for which $(\mathcal{D}, w)$ is exact. The precision space of $(\mathcal{D}, w)$ is the $\mathbb{C}$-vector $\operatorname{space} \operatorname{Span}\left(\mathcal{B}_{\mathcal{D}, w}\right)$ generated by $\mathcal{B}_{\mathcal{D}, w}$.

In the univariate case the notion of precision degree of a quadrature rule is used. It is the maximal degree of the elements of $\mathcal{P}$ on which the quadrature rule is exact, see e.g. [7]. For example, if $\mathcal{D}$ is a subset of $\mathbb{R}$ of cardinality $n$ and if $\mathcal{P}$ is the interpolation space generated by $\left\{1, x, x^{2}, \ldots, x^{n-1}\right\}$, the precision degree is $2 n-1$. So that the precision space of Gaussian quadrature rule is generated by $\mathcal{B}_{\mathcal{D}, w}=\left\{1, x, x^{2}, \ldots, x^{2 n-1}\right\}$.

Generalising the notion of precision degree to the multivariate case, we define the precision degree of $(\mathcal{D}, w)$ as the $\max _{z^{\alpha} \in \mathcal{B}_{\mathcal{D}, w}}\left\{\sum_{i=1}^{k} \alpha_{i}\right\}$.

\section{Weights for points in $\mathbb{C}$-vector space with basis $S$}

Let $\mathcal{D}$ be a set of $n$ nodes in $\mathbb{C}^{k}$, let $S \subset \mathbb{T}$ be a set of monomials in $\mathbb{C}\left[z_{1}, \ldots, z_{k}\right]$ and let $\mathcal{P}=\operatorname{Span}_{\mathbb{C}}(S)$ be the $\mathbb{C}$-vector space of polynomials in $\mathbb{C}\left[z_{1}, \ldots, z_{k}\right]$ generated by $S$ such that $(\mathcal{D}, \mathcal{P})$ is correct.

The following proposition gives the vector of weights $w_{S}$ that makes $(\mathcal{D}, w)$ exact on $\mathcal{P}$, that is the weights $w_{S}$ of the interpolatory cubature rule on $\mathcal{P}$.

Proposition 3.1. Let $\mathcal{P}$ be a $\mathbb{C}$-vector space with basis $S$ with $\# S=\# \mathcal{D}$, and let $(\mathcal{D}, \mathcal{P})$ be correct. Let $X_{\mathcal{D}, S}=[s(d)]_{d \in \mathcal{D}, s \in S}$ be the evaluation matrix of the elements of $S$ over $\mathcal{D}$. The cubature rule $\left(\mathcal{D}, w_{S}\right)$ is exact on $\mathcal{P}$ if and only if

$$
w_{S}=\left(X_{\mathcal{D}, S}^{t}\right)^{-1}\left[\int_{\mathbb{C}^{k}} s d \lambda\right]_{s \in S} .
$$

where $X_{\mathcal{D}, S}^{t}$ is the transposed matrix of $X_{\mathcal{D}, S}$. Furthermore, the weights $w_{S}$ are unique.

Proof. Each $p \in \mathcal{P}$ can be written uniquely as

$$
p=\sum_{s \in S} c_{s} s, \text { for } c_{s} \in \mathbb{C},
$$

and so

$$
[p(d)]_{d \in \mathcal{D}}=X_{\mathcal{D}, S}\left[c_{s}\right]_{s \in S},
$$

that is

$$
\left[c_{s}\right]_{s \in S}=X_{\mathcal{D}, S}^{-1}[p(d)]_{d \in \mathcal{D}} .
$$


Claudia Fassino, Eva Riccomagno, Maria Piera Rogantin / J. Alg. Stat., 10, No.1 (2019), pp.115-127 118

It follows that, for each $p \in \mathcal{P}$,

$$
\begin{aligned}
\int_{\mathbb{C}^{k}} p d \lambda & =\int_{\mathbb{C}^{k}} \sum_{s \in S} c_{s} s d \lambda=\sum_{s \in S} c_{s} \int_{\mathbb{C}^{k}} s d \lambda=\left[\int_{\mathbb{C}^{k}} s d \lambda\right]_{s \in S}^{t}\left[c_{s}\right]_{s \in S} \\
& =\left[\int_{\mathbb{C}^{k}} s d \lambda\right]_{s \in S}^{t} X_{\mathcal{D}, S}^{-1}[p(d)]_{d \in \mathcal{D}}
\end{aligned}
$$

The cubature rule $\left(\mathcal{D}, w_{S}\right)$ is exact on $\mathcal{P}$ if and only if $\int_{\mathbb{C}^{k}} p d \lambda=w_{S}^{t}[p(d)]_{d \in \mathcal{D}}$ for each $p \in \mathcal{P}$, that is if and only if

$$
\left[\int_{\mathbb{C}^{k}} s d \lambda\right]_{s \in S}^{t} X_{\mathcal{D}, S}^{-1}[p(d)]_{d \in \mathcal{D}}=w_{S}^{t}[p(d)]_{d \in \mathcal{D}}
$$

or, equivalently,

$$
w_{S}=\left(X_{\mathcal{D}, S}^{t}\right)^{-1}\left[\int_{\mathbb{C}^{k}} s d \lambda\right]_{s \in S}+\rho
$$

where $\rho$ is orthogonal to each evaluation vector $[p(d)]_{d \in \mathcal{D}}$. In particular, $\rho$ is orthogonal to $[s(d)]_{d \in \mathcal{D}}, s \in S$, that is to the columns of $X_{\mathcal{D}, S}$. Since $X_{\mathcal{D}, S}$ is a square non singular matrix, $\rho$ is the null vector and so the vector $w_{S}$ of the weights of the cubature rule $(\mathcal{D}, \operatorname{Span}(S))$ is unique.

Remark 3.2. The weights $w_{S}$ do not change, if a different basis $T$ for the $\mathbb{C}$-vector space $\mathcal{P}=\operatorname{Span}(S)$ is chosen. Each monomial $t \in T$ can be expressed as $t=\sum_{s \in S} m_{t, s} s$, and so, denoting by $M=\left[m_{t, s}\right]_{t \in T, s \in S}$, we have, by linearity,

$$
\left[\int_{\mathbb{C}^{k}} t d \lambda\right]_{t \in T}=M\left[\int_{\mathbb{C}^{k}} s d \lambda\right]_{s \in S} .
$$

Moreover, the evaluation matrix $X_{\mathcal{D}, T}$ of the elements of $T$ over $\mathcal{D}$ can be written as $X_{\mathcal{D}, T}=X_{\mathcal{D}, S} M^{t}$. Let $w_{T}$ be the weights computed using $\mathcal{D}$ and $T$. From Proposition 3.1 we have

$$
\begin{aligned}
w_{T} & =\left(X_{\mathcal{D}, T}^{t}\right)^{-1}\left[\int_{\mathbb{C}^{k}} t d \lambda\right]_{t \in T}=\left(M X_{\mathcal{D}, S}^{t}\right)^{-1} M\left[\int_{\mathbb{C}^{k}} s d \lambda\right]_{s \in S} \\
& =\left(X_{\mathcal{D}, S}^{t}\right)^{-1}\left[\int_{\mathbb{C}^{k}} s d \lambda\right]_{s \in S}=w_{S}
\end{aligned}
$$

\section{4. $\mathbb{C}$-Fractional factorial designs}

In this section we consider interpolatory cubature rules with set of nodes $\mathcal{D}$, whose elements are $k$-uple of $m$-th roots of the unit, and with interpolatory space $\mathcal{P}$, the $\mathbb{C}$-vector space generated by a monomial set $S \subset \mathbb{T}$, such that the pair $(\mathcal{D}, \mathcal{P})$ is correct.

We briefly recall some topics about of the roots of the unity. Let $m \in \mathbb{Z}_{\geq 0}$ and $\Omega_{m}=\left\{\omega_{0}, \ldots, \omega_{m-1}\right\}$ be the set of the $m$-th roots of the unity, $\omega_{j}=\exp (-\mathbf{i}(2 \pi / m) j)$, where $\mathbf{i}=\sqrt{-1}$ is the imaginary unity. 
Claudia Fassino, Eva Riccomagno, Maria Piera Rogantin / J. Alg. Stat., 10, No.1 (2019), pp.115-127 119

Denoting, for $j \in \mathbb{Z}$, by $[j]_{m}$ the residue of $j \bmod m$ and by $\bar{j}$ the class $[m-j]_{m}$ we have that, given $c \in \mathbb{Z}$ and $\omega_{j}, \omega_{i} \in \Omega_{m}$, it holds $\omega_{j}^{c}=\omega_{[c j]_{m}}, \omega_{i} \omega_{j}=\omega_{[i+j]_{m}}$, and the complex conjugate of $\omega_{j}$ is $\overline{\omega_{j}}=\omega_{\bar{j}}$. Furthermore, we denote by $\mathbb{Z}_{m}$ the set of all congruence classes of the integers for a modulus $m$, and by $\mathbb{Z}_{m}^{k}$ its cartesian product.

We consider a set of $n$ nodes $\mathcal{D}$ contained in $\Omega_{m}^{k} \subset \mathbb{C}^{k}$. Let $f$ be the indicator function of $\mathcal{D}$ over $\Omega_{m}^{k}$, defined as $f(d)=1$, for $d \in \mathcal{D}$, and $f(d)=0$, for $d \in \Omega_{m}^{k} \backslash \mathcal{D}$.

Let $S_{m}=\left\{z^{\alpha}: \alpha \in \mathbb{Z}_{m}^{k}\right\}$ be the monomial basis of the $\mathbb{C}$-vector space which is isomorphic to the quotient space $\mathbb{C}\left[z_{1}, \ldots, z_{k}\right] / I\left(\Omega_{m}^{k}\right)$. As presented in [14], by interpolating the values $[f(d)]_{d \in \Omega_{m}^{k}}$ with polynomials in $\operatorname{Span}\left(S_{m}\right)$, we obtain a representation of the indicator function $f$ as follows

$$
f=\sum_{\alpha \in \mathbb{Z}_{m}^{k}} b_{\alpha} z^{\alpha} \quad \text { where } \quad b_{\alpha}=\frac{1}{m^{k}} \sum_{d \in \mathcal{D}} z^{\bar{\alpha}}(d)
$$

and, since $\alpha=\left[\alpha_{1}, \ldots, \alpha_{k}\right] \in \mathbb{Z}_{m}^{k}, \bar{\alpha}=\left[m-\alpha_{1}, \ldots, m-\alpha_{k}\right]$.

Special subsets $\mathcal{D}$ of $\Omega_{m}^{k}$ are the regular fractions, where the evaluation of two monomials $z^{\alpha}$ and $z^{\beta}, \alpha$ and $\beta$ in $\mathbb{Z}_{m}^{k}$, are orthogonal vectors or proportional to $1_{n}$ vectors. The absolute values of the non-null coefficients of the indicator function of a regular fraction are all equal to $\# \mathcal{D} / \mathrm{m}^{k}$.

Let $\left(\mathcal{D}, w_{S}\right)$ be the cubature rule with nodes $\mathcal{D} \subset \Omega_{m}^{k}, S \subset S_{m}$ and weights $w_{S}=$ $\left(X_{\mathcal{D}, S}^{t}\right)^{-1}\left[\int_{\mathbb{C}^{k}} s d \lambda\right]_{s \in S}$.

In the next we consider non-negative and normalized weights, i.e. such that $w_{S}^{t} 1_{n}=1$, where $1_{n}$ is a $n$-vector with elements equal to 1 , and the weights are all equal, that is $w_{S}=\frac{1}{n} 1_{n}$.

\subsection{Equal weights}

If the weights are non-negative, normalized and all equal, then $w=\frac{1}{n} 1_{n}$. The following theorem characterises a cubature rule with equal weights using the indicator function of $\mathcal{D}$.

Theorem 4.1. Let $\mathcal{D} \subset \Omega_{m}^{k}$ be a set of $n$ nodes and let $f$ its indicator function, as in $E q$. (1). Let $S \subset S_{m}$ be a monomial set such that the pair $(\mathcal{D}, \operatorname{Span}(S))$ is correct and let $w_{S}$ as in Proposition 3.1.

Let $A$ be the set:

$$
A=\left\{z^{\alpha} \in \mathbb{T}, \alpha \in \mathbb{Z}_{\geq 0}^{k} \mid \int_{\mathbb{C}^{k}} z^{\alpha} d \lambda=\frac{m^{k}}{n} b_{\bar{\alpha}}\right\}
$$

Then $w_{S}=\frac{1}{n} 1_{n}$ if and only if $S \subseteq A$.

Proof. From Proposition 3.1 and Eq. (1) (restricted to the $\alpha$ such that $z^{\alpha} \in S$ ) it holds:

$$
w_{S}=\left(X_{\mathcal{D}, S}^{t}\right)^{-1}\left[\int_{\mathbb{C}^{k}} z^{\alpha} d \lambda\right]_{z^{\alpha} \in S} \quad \text { and } \quad\left[b_{\bar{\alpha}}\right]_{z^{\alpha} \in S}=\frac{1}{m^{k}} X_{\mathcal{D}, S}^{t} 1_{n}
$$


Claudia Fassino, Eva Riccomagno, Maria Piera Rogantin / J. Alg. Stat., 10, No.1 (2019), pp.115-127 120

If $w_{S}=\frac{1}{n} 1_{n}$ then

$$
\left[\int_{\mathbb{C}^{k}} z^{\alpha} d \lambda\right]_{z^{\alpha} \in S}=\frac{1}{n} X_{\mathcal{D}, S}^{t} 1_{n}=\frac{m^{k}}{n}\left[b_{\bar{\alpha}}\right]_{z^{\alpha} \in S}
$$

so that $S \subseteq A$.

Vice-versa, if $S \subseteq A$, for $z^{\alpha} \in S$, it holds:

$$
\left[b_{\bar{\alpha}}\right]_{z^{\alpha} \in S}=\frac{n}{m^{k}}\left[\int_{\mathbb{C}^{k}} z^{\alpha} d \lambda\right]_{z^{\alpha} \in S}=\frac{n}{m^{k}} X_{\mathcal{D}, S}^{t} w_{S}
$$

and, from $\left[b_{\bar{\alpha}}\right]_{z^{\alpha} \in S}=\frac{1}{m^{k}} X_{\mathcal{D}, S}^{t} 1_{n}$, it follows $w_{S}=\frac{1}{n} 1_{n}$, being $X_{\mathcal{D}, S}$ invertible.

Given a set $\mathcal{D} \subseteq \Omega_{m}^{k}$ with $n$ points, Theorem 4.1 suggests an algorithm for finding, if there exists, a cubature rule with nodes $\mathcal{D}$ and equal weights. Fix $X^{(0)}=1_{n}$ and $S=\{1\}$. At the $r$-th step an element $z^{\alpha}$ of $A \cap\left\{z^{\alpha} \in \mathbb{T}, \alpha \in \mathbb{Z}_{m}^{k}\right\}$ is considered. If the vector $v=\left[z^{\alpha}(d)\right]_{d \in \mathcal{D}}$ is such that the matrix $\left[X^{(r-1)}, v\right]$ has full rank, then $v$ is added to the matrix $X^{(r-1)}$ for obtaining the new matrix $X^{(r)}=\left[X^{(r-1)}, v\right]$ and $z^{\alpha}$ is added to $S$. Otherwise a different element of $A \cap\left\{z^{\alpha} \in \mathbb{T}, \alpha \in \mathbb{Z}_{m}^{k}\right\}$ is considered.

The algorithm stops when a square non singular matrix $X^{(n-1)}$ is computed or if all the elements of $A \cap\left\{z^{\alpha} \in \mathbb{T}, \alpha \in \mathbb{Z}_{m}^{k}\right\}$ are analysed. In the former case the basis $S$ is such that the cubature rule $\left(\mathcal{D}, w_{S}\right)$ has equal weights. In the latter case there not exists any basis such that the associated cubature rule with node $\mathcal{D}$ has equal weights. Notice that the algorithm stops because the elements of $A \cap\left\{z^{\alpha} \in \mathbb{T}, \alpha \in \mathbb{Z}_{m}^{k}\right\}$ are finite.

The following theorem characterises the precision basis of cubature rules with equal weights.

Theorem 4.2. Let $\mathcal{D} \subset \Omega_{m}^{k}$ be a set of $n$ nodes and let $f$ its indicator function, as in $E q$. (1). Let $S \subset S_{m}$ be a monomial set such that the pair $(\mathcal{D}, \operatorname{Span}(S))$ is correct and let $w_{S}$ as in Proposition 3.1.

If $w_{S}=\frac{1}{n} 1_{n}$ then the set $A$ defined in Theorem 4.1 is the precision basis $\mathcal{B}$ for $\left(\mathcal{D}, w_{S}\right)$.

Proof. From Eq. (1), for each $\alpha \in[0, \ldots, m-1]^{k}, b_{\bar{\alpha}}=\frac{1}{m^{k}} \sum_{d \in \mathcal{D}} z^{\alpha}(d)$, that is $\sum_{d \in \mathcal{D}} z^{\alpha}(d)=m^{k} b_{\bar{\alpha}}$

For each $\alpha \in \mathbb{Z}_{\geq 0}^{k}$ it holds $z^{\alpha}(d)=z^{[\alpha]_{m}}(d)$. Then:

$$
\sum_{d \in \mathcal{D}} z^{\alpha}(d)=\sum_{d \in \mathcal{D}} z^{[\alpha]_{m}}(d)=m^{k} b_{\bar{\alpha}}
$$

Let $w=\frac{1}{n} 1_{n}$ be the weights. Then the precision space for $\left(\mathcal{D}, \frac{1}{n} 1_{n}\right)$ is the largest set of monomials for which

$$
\int_{\mathbb{C}^{k}} z^{\alpha} d \lambda=\frac{1}{n} \sum_{d \in \mathcal{D}} z^{\alpha}(d) \quad \text { i.e. } \quad \int_{\mathbb{C}^{k}} z^{\alpha} d \lambda=\frac{m^{k}}{n} b_{\bar{\alpha}}
$$


Claudia Fassino, Eva Riccomagno, Maria Piera Rogantin / J. Alg. Stat., 10, No.1 (2019), pp.115-127 121 that is $A$.

The following result describes the behaviour of a cubature rule with equal weights, when it is applied to the integral of monomials of the form $z^{\alpha} \bar{z}^{\beta}$. The connection to evaluation of moments of a distribution is evident.

Theorem 4.3. Let $\left(\mathcal{D}, w_{s}\right)$ be a cubature rule with $w_{s}=\frac{1}{n} 1_{n}$. The cubature rule $\left(\mathcal{D}, w_{s}\right)$ is exact for $z^{\alpha}$

1. if and only if $\left(\mathcal{D}, w_{s}\right)$ is exact for $\bar{z}^{\alpha}$.

2. if and only if $\left(\mathcal{D}, w_{s}\right)$ is exact for $z^{\alpha+\gamma} \bar{z}^{\gamma}$ for each $\gamma \in \mathbb{Z}^{k}$ such that $\int_{\mathbb{C}^{k}} z^{\alpha+\gamma} \bar{z}^{\gamma} d \lambda=$ $\int_{\mathbb{C}^{k}} z^{\alpha} d \lambda$.

Proof.

1. If $\left(\mathcal{D}, w_{s}\right)$ is exact for $z^{\alpha}$, then

$$
\int_{\mathbb{C}^{k}} z^{\alpha} d \lambda=\frac{1}{n} \sum_{d \in \mathcal{D}} z^{\alpha}(d)
$$

and so

$$
\int_{\mathbb{C}^{k}} \bar{z}^{\alpha} d \lambda=\int_{\mathbb{C}^{k}} \overline{z^{\alpha}} d \lambda=\overline{\int_{\mathbb{C}^{k}} z^{\alpha} d \lambda}=\frac{1}{n} \overline{\sum_{d \in \mathcal{D}} z^{\alpha}(d)}=\frac{1}{n} \sum_{d \in \mathcal{D}} \overline{z^{\alpha}(d)}=\frac{1}{n} \sum_{d \in \mathcal{D}} \bar{z}^{\alpha}(d)
$$

and we conclude that $\left(\mathcal{D}, w_{s}\right)$ is exact for $\bar{z}^{\alpha}$. The vice-versa is analogous.

2. Let $\left(\mathcal{D}, w_{s}\right)$ be exact for $z^{\alpha}$. By assumptions $\int_{\mathbb{C}^{k}} z^{\alpha+\gamma} \bar{z}^{\gamma} d \lambda=\int_{\mathbb{C}^{k}} z^{\alpha} d \lambda$ and so

$$
\begin{aligned}
\int_{\mathbb{C}^{k}} z^{\alpha+\gamma} \bar{z}^{\gamma} d \lambda & =\int_{\mathbb{C}^{k}} z^{\alpha} d \lambda=\frac{1}{n} \sum_{d \in \mathcal{D}} z^{\alpha}(d)=\frac{1}{n} \sum_{d \in \mathcal{D}}\left|z^{\gamma}(d)\right|^{2} z^{\alpha}(d) \\
& =\frac{1}{n} \sum_{d \in \mathcal{D}} z^{\alpha+\gamma}(d) \bar{z}^{\gamma}(d),
\end{aligned}
$$

where the third equality is due to the fact that $\left|z^{\gamma}(d)\right|^{2}=1$. We conclude that $\left(\mathcal{D}, w_{s}\right)$ is exact for $z^{\alpha+\gamma} \bar{z}^{\gamma}$. Furthermore, from item 1 it follows that, since the cubature rule is exact for $z^{\alpha+\gamma} \bar{z}^{\gamma}$, it is also exact for $\overline{z^{\alpha+\gamma} \bar{z}^{\gamma}}$, that is for $\bar{z}^{\alpha+\gamma} z^{\gamma}$.

The vice-versa is analogous.

\subsection{Gaussian distribution}

In this section we characterise the cubature rules $(\mathcal{D}, S)$ with equal weights and the Gaussian distribution. First of all, we present some results about integration with respect to the Gaussian measure. 
Claudia Fassino, Eva Riccomagno, Maria Piera Rogantin / J. Alg. Stat., 10, No.1 (2019), pp.115-127 122

\subsubsection{Gaussian measure}

Let $Z^{t}=\left(Z_{1}, \ldots, Z_{p}\right)$ be a $p$-variate Gaussian complex random variable. Let $Z_{k}=$ $X_{k}+\mathrm{i} Y_{k}, k=1, \ldots, p$, then the vector of real and imaginary parts $\left(X_{1}, Y_{1}, \ldots, X_{p}, Y_{p}\right)$ is a $2 p$-variate Gaussian real random vector. We assume the following relations among the expected values of the real and imaginary parts of the $Z$ variables.

$$
\begin{array}{cc}
E\left(X_{k}\right)=E\left(Y_{k}\right)=0 & \\
E\left(X_{j} X_{j}\right)=E\left(Y_{j} Y_{j}\right)=\frac{\sigma_{j}^{2}}{2} & E\left(X_{j} Y_{j}\right)=0 \\
E\left(X_{j} X_{k}\right)=E\left(Y_{j} Y_{k}\right)=\frac{\alpha_{j k}}{2} \text { for } j \neq k & E\left(X_{j} Y_{k}\right)=-E\left(X_{k} Y_{j}\right)=-\frac{\beta_{j k}}{2} \text { for } j>k
\end{array}
$$

for $j, k=1, \ldots, p$.

We denote by $\Sigma$ the matrix $E\left(Z \bar{Z}^{t}\right)=\left[E\left(Z_{j} \bar{Z}_{k}\right)\right]_{j, k=1, \ldots, p}$. Then, from the previous conditions,

$$
\Sigma_{j k}=E\left(Z_{j} \bar{Z}_{k}\right)= \begin{cases}\sigma_{k}^{2} & \text { if } j=k \\ \alpha_{j k}+\mathbf{i} \beta_{j k} & \text { if } j<k \\ \alpha_{j k}-\mathbf{i} \beta_{j k} & \text { if } j>k\end{cases}
$$

The probability density function of the zero mean $p$-variate complex Gaussian distribution is given by (see e.g. [8])

$$
p(z)=\frac{1}{\pi^{p} \operatorname{det}(\Sigma)} \exp \left(-\bar{z}^{t} \Sigma^{-1} z\right)
$$

where $z=\left[z_{1}, \ldots, z_{p}\right]^{t}$.

We consider the complex measure $\nu$ such that $d \nu=p(z) d \mu$, where $\mu$ is the $\sigma$-finite measure of $\mathbb{C}^{p}$ identifiable with the Lebesgue measure of $\mathbb{R}^{2 p}$, and so from the results presented in Appendix A, since $z_{k}=x_{k}+\mathbf{i} y_{k}, k=1 \ldots p$, we have $\int_{\mathbb{C}^{p}} f(z) d \nu=\int_{\mathbb{C}^{p}} f(z) p(z) d \mu=$ $\int_{\mathbb{R}^{2 p}} f(x, y) p(x, y) d x d y$, where $x=\left[x_{1}, \ldots, x_{p}\right]^{t}$ and $y=\left[y_{1}, \ldots, y_{p}\right]^{t}$.

We denote by $\nu\left(n_{1}, m_{1}, \ldots, n_{p}, m_{p}\right)$ the moment:

$\nu\left(n_{1}, m_{1}, \ldots, n_{p}, m_{p}\right)=\frac{1}{\pi^{p} \operatorname{det}(\Sigma)} \int_{\mathbb{C}^{p}} z_{1}^{n_{1}} \bar{z}_{1}^{m_{1}} z_{2}^{n_{2}} \bar{z}_{2}^{m_{2}} \cdots z_{p}^{n_{p}} \bar{z}_{p}^{m_{p}} \exp \left(-\bar{z}^{t} \Sigma^{-1} z\right) d z_{1} \cdots d z_{p}$

In the vector $\left(n_{1}, m_{1}, \ldots, n_{p}, m_{p}\right)$, consisting of the exponents of the moments, the $n_{j}$ indices are in odd entries and refer to $Z_{j}$ while the $m_{j}$ indices are in even entries and refer to $\bar{Z}_{j}$.

In [4] the following theorem on the null moments is shown.

Theorem 4.4 (Null moments). If no variable $Z_{j}, j=1, \ldots, p$, is independent from all the others, then $\nu(\alpha)$ is null if:

$$
\sum_{j=1}^{p} n_{j} \neq \sum_{j=1}^{p} m_{j}
$$


Claudia Fassino, Eva Riccomagno, Maria Piera Rogantin / J. Alg. Stat., 10, No.1 (2019), pp.115-127 123

From Theorem 4.4 we obtain the value of the integral of the monomials in $\mathbb{T}$ with respect to the Gaussian distribution.

Corollary 4.5. Let $z^{\alpha}=z_{1}^{n_{1}} \cdots z_{p}^{n_{p}}$ a monomial in $\mathbb{T}$. Then

$$
\left\{\begin{array}{l}
\int_{\mathbb{C}^{p}} z^{\alpha} p(z) d z=0 \quad \text { if there exists } r \in\{1, \ldots, p\} \text { s.t. } n_{r} \neq 0 \\
\int_{\mathbb{C}^{p}} z^{\alpha} p(z) d z=1 \quad \text { if } n_{1}=\cdots=n_{p}=0
\end{array}\right.
$$

Proof. In this case $m_{1}=\cdots=m_{p}=0$, and so, if there exists an exponent $n_{r} \neq 0$, from Theorem 4.4 we have $\nu\left(n_{1}, 0, \ldots, n_{p}, 0\right)=\frac{1}{\pi^{p} \operatorname{det}(\Sigma)} \int_{\mathbb{C}^{p}} z_{1}^{n_{1}} \cdots z_{p}^{n_{p}} p(z) d z_{1} \cdots d z_{p}=0$ and the first part of the thesis follows. The second part is an obvious result.

\subsubsection{Cubature rules with equal weights for Gaussian distribution}

Let $\nu$ be the Gaussian distribution. Since, in this case $\int_{\mathbb{C}^{k}} z^{\alpha} d \nu=0$, if $\alpha \neq(0, \ldots, 0)$, Theorem 4.1 can be reformulated as follows.

Theorem 4.6. Let $\nu$ be the Gaussian distribution. Let $\mathcal{D} \subset \Omega_{m}^{k}$ be a set of $n$ nodes and let $f$ its indicator function, as in Eq. (1). Let $S \subset S_{m}$ be a monomial set such that the pair $(\mathcal{D}, \operatorname{Span}(S))$ is correct and let $w_{S}$ as in Proposition 3.1. Then $w_{S}=\frac{1}{n} 1_{n}$ if and only if $S \cap \operatorname{Supp}(f)=\{1\}$.

Proof. From Theorem 4.1 we have that $w_{S}=\frac{1}{n} 1_{n}$ if and only if $S \subset A$, where

$$
A=\left\{z^{\alpha} \in \mathbb{T}, \alpha \in \mathbb{Z}_{\geq 0}^{k} \mid \int_{\mathbb{C}^{k}} z^{\alpha} d \nu=\frac{m^{k}}{n} b_{\bar{\alpha}}\right\},
$$

and so we first describe $A$ for the Gaussian distribution.

When $\alpha=[0, \ldots, 0]$ we have that $\int z^{\alpha} d \nu=1$ and $b_{\bar{\alpha}}=\frac{n}{m^{k}}$ and so 1 belongs to $A$. Furthermore, if $\alpha \neq[0, \ldots, 0]$, then $\int_{\mathbb{C}^{k}} z^{\alpha} d \nu=0$, and so the set $A$ is given by

$$
A=\{1\} \cup\left\{z^{\alpha} \in \mathbb{T}, \alpha \in \mathbb{Z}_{>0}^{k} \mid b_{\bar{\alpha}}=0\right\}=\{1\} \cup\left\{z^{\alpha} \in \mathbb{T}, \alpha \in \mathbb{Z}_{>0}^{k} \mid b_{[\alpha]_{m}}=0\right\}
$$

since $b_{[\alpha]_{m}}=\overline{b_{\bar{\alpha}}}$. From Equation (1), we have that $b_{[\alpha]_{m}}=0$ if and only if $z^{[\alpha]_{m}} \notin \operatorname{Supp}(f)$, and thus

$$
A=\{1\} \cup\left\{z^{\alpha} \in \mathbb{T}, \alpha \in \mathbb{Z}_{>0}^{k}, z^{[\alpha]_{m}} \notin \operatorname{Supp}(f)\right\} .
$$

We conclude that $S \subset A$ if and only if $S \cap \operatorname{Supp}(f)=\{1\}$.

The following theorem characterises the precision basis of a cubature rule with equal weights, with respect to the gaussian distribution.

Corollary 4.7. Let $\nu$ be the Gaussian distribution. Let $\mathcal{D} \subset \Omega_{m}^{k}$ be a set of $n$ nodes and let $f$ its indicator function, as in Eq. (1). Let $S \subset S_{m}$ be a monomial set such that the pair $(\mathcal{D}, \operatorname{Span}(S))$ is correct and let $w_{S}$ as in Proposition 3.1. If $w_{S}=\frac{1}{n} 1_{n}$ then $\{1\} \cup\left\{z^{\alpha} \in \mathbb{T} \mid z^{[\alpha]_{m}} \notin \operatorname{Supp}(f)\right\}$ is the precision basis $\mathcal{B}$ for $\left(\mathcal{D}, w_{S}\right)$. 
Claudia Fassino, Eva Riccomagno, Maria Piera Rogantin / J. Alg. Stat., 10, No.1 (2019), pp.115-127 124

Given a set of nodes $\mathcal{D}$, it is possible to check if there exists a basis $S$ so that the corresponding cubature rule $(\mathcal{D}, S)$ has equal weights in an easier way than in the general case, since it is sufficient to consider only monomials $t \in \mathbb{Z}_{m}^{k} \backslash \operatorname{Supp}(f)$, as Corollary 4.7 shows that $S \cap \operatorname{Supp}(f)=\{1\}$ in order to have equal weights.

Proposition 4.8. Let $\nu$ be the Gaussian distribution. If $\mathcal{D}$ is a regular fraction, for each basis $S$ such that the pair $(\mathcal{D}, \operatorname{Span}(S))$ is correct, the corresponding cubature rule has equal weights.

Proof. If $\mathcal{D}$ is a regular fraction, for each monomial $s \in \mathbb{T}$, the vector $[s(d)]_{d \in \mathcal{D}}$ is equal to $\gamma 1_{n}$, for a given $\gamma \in \mathbb{C}$, or orthogonal to $1_{n}$. Since $X_{\mathcal{D}, S}$ is a non singular matrix whose first column is the vector $1_{n}$, for each monomial basis $S=\left\{z^{\alpha}\right\}$, the columns of $X_{\mathcal{D}, S}$, except the first one, are orthogonal to $1_{n}$. It follows that $\Sigma_{d \in \mathcal{D}} z^{\alpha}(d)=0$ for each $z^{\alpha} \in S \backslash\{1\}$ and so $z^{\alpha} \notin \operatorname{Supp}(f)$. We conclude that, if $\mathcal{D}$ is a regular fraction, $S \cap \operatorname{Supp}(f)=\{1\}$ for each possible basis $S$ and so the corresponding cubature rule has equal weights.

The following example shows that a cubature rule can have equal weights even if $\mathcal{D}$ is not a regular fraction of $\Omega_{m}^{k}$.

Example 4.9. Let $\mathbb{C}\left[z_{1}, \ldots, z_{4}\right]$ be the polynomial ring with indeterminates $z_{1}, \ldots, z_{4}$ and let $\mathcal{D}$ be the set of nodes contained in $\Omega_{2}^{4}$,

$$
\begin{aligned}
\mathcal{D}=\{(1,1,1,1),(1,1,-1,1),(1,-1,1,-1),(1,-1,-1,-1), & \\
& (-1,1,1,1),(-1,-1,-1,1),(-1,-1,-1,-1),(-1,1,1,-1)\}
\end{aligned}
$$

whose indicator function is $f=\left(2+z_{2} z_{3}+z_{2} z_{4}-z_{1} z_{2} z_{3}+z_{1} z_{2} z_{4}\right) / 4$. The set $\mathcal{D}$ is not a regular fraction since the coefficients of its indicator function are not all equal.

Given the monomial set $S=\left\{1, z_{1}, z_{2}, z_{3}, z_{4}, z_{1} z_{2}, z_{3} z_{4}, z_{1} z_{3} z_{4}\right\}$, the weights $w_{S}$ are the solution of the linear system $X_{\mathcal{D}, S}^{t} w_{S}=\left[\int s d \nu\right]_{s \in S}$, where $\int s d \nu=0$ if $s \neq 1$ and $\int s d \nu=1$ if $s=1$. Since the columns of the matrix $X_{\mathcal{D}, S}$, except the first one, are orthogonal to $1_{8}$, the vector $w_{S}=\frac{1}{8} 1_{8}$ is the solution of the previous linear system, even if the set of nodes is not a regular fraction. However, we observe that $\mathcal{D}$ is the union of two disjoint regular fractions of indicator functions $f_{1}=\left(1-z_{1}+z_{2} z_{3}-z_{1} z_{2} z_{3}\right) / 4$ and $f_{2}=\left(1+z_{1}+z_{2} z_{4}+z_{1} z_{2} z_{4}\right) / 4$ respectively.

The following example illustrates a cubature rule with equal weights and its precision basis.

Example 4.10. Let $k=2, m=4$ and let $\omega_{0}=1, \omega_{1}=\mathbf{i}, \omega_{2}=-1$ and $\omega_{3}=-\mathbf{i}$ be the fourth root of the unit. In this case. Let $\mathcal{D}=\{(1,1),(\mathbf{i},-\mathbf{i}),(-1, \mathbf{i}),(-\mathbf{i},-1)\} \subset \Omega_{4}^{2}$ be the set of nodes with indicator function

$$
f=\frac{1}{8}\left(2+z_{1} z_{2}+(1+\mathbf{i}) z_{1} z_{2}^{2}+(1-\mathbf{i}) z_{1}^{2} z_{2}-\mathbf{i} z_{1} z_{2}^{3}+\mathbf{i} z_{1}^{3} z_{2}+(1+\mathbf{i}) z_{1}^{2} z_{2}^{3}+(1-\mathbf{i}) z_{1}^{3} z_{2}^{2}+z_{1}^{3} z_{2}^{3}\right)
$$


Claudia Fassino, Eva Riccomagno, Maria Piera Rogantin / J. Alg. Stat., 10, No.1 (2019), pp.115-127 125

Given the monomial set $S=\left\{1, z_{2}, z_{1}, z_{2}^{3}\right\}$, we have $S \cap \operatorname{Supp}(f)=\{1\}$ and so the weights of the cubature rule $(\mathcal{D}, S)$ are $w_{d}=\frac{1}{4}$, for all $d \in \mathcal{D}$. From Corollary 4.7 it follows that the precision basis is

$$
\mathcal{B}_{\mathcal{D}, S}=\{1\} \cup\left\{z^{\alpha} \in \mathbb{T},[\alpha]_{4} \in\{(0,1),(1,0),(0,2),(2,0),(0,3),(3,0),(2,2)\}\right\} .
$$

From Theorem 4.3 it follows that the cubature rule is also exact for $\left\{\bar{z}^{\alpha}, z^{\alpha} \in \mathcal{B}_{\mathcal{D}, S}\right\}$. Furthermore, since for each $\alpha \neq(0, \ldots, 0)$ and for each $\gamma \in \mathbb{Z}_{\geq 0}^{4}$ we have $\int_{\mathbb{C}^{k}} z^{\alpha} d \nu=0=$ $\int_{\mathbb{C}^{k}} z^{\alpha+\gamma} \bar{z}^{\gamma} d \nu$, the cubature rule is also exact for $\left\{\bar{z}^{\alpha+\gamma} z^{\gamma}, z^{\alpha+\gamma} \bar{z}^{\gamma}, z^{\alpha} \in \mathcal{B}_{\mathcal{D}, S}, \gamma \in \mathbb{Z}_{\geq 0}^{4}\right\}$.

The following example shows the case of a set of nodes $\mathcal{D}$ which does not generate any cubature rule with equal weights.

Example 4.11. Let $k=2, m=3$ and let $\omega_{0}=1, \omega_{1}=\cos (2 \pi / 3)+\mathbf{i} \sin (2 \pi / 3)$ and $\omega_{2}=\bar{\omega}_{1}$ be the third root of the unit.

Let $\mathcal{D}=\left\{\left(1, \omega_{2}\right),\left(\omega_{2}, \omega_{1}\right)\right\}$ be the set of nodes with indicator function

$$
f=\frac{1}{9}\left(2-z_{2}-\omega_{2} z_{1}-z_{2}^{2}-\omega_{2} z_{1} z_{2}-\omega_{1} z_{1}^{2}+2 \omega_{2} z_{1} z_{2}^{2}+2 \omega_{1} z_{1}^{2} z_{2}-\omega_{1} z_{1}^{2} z_{2}^{2}\right) .
$$

Since $\operatorname{Supp}(f)=\left\{z^{\alpha} \in \mathbb{T}, \alpha \in \mathbb{Z}_{3}^{2}\right\}$, there not exist a monomial basis $S \subset\left\{z^{\alpha} \in \mathbb{T}, \alpha \in \mathbb{Z}_{3}^{2}\right\}$ such that $S \cap \operatorname{Supp}(f)=\{1\}$ and so there not exist a cubature rule $(\mathcal{D}, S)$ with equal weights.

The previous examples seem to suggest that the existence of a cubature rule with equal weights is highly related to some partition of the set of nodes in regular fractions. This topic is subject of ongoing research.

\section{Appendix A. Complex integration}

Let $\mathcal{M}$ be a $\sigma$-algebra in a set $X$ and let $\left\{E_{k}\right\}$ be a countable partition of $E$, that is $E=\cup_{k} E_{k}$ and $E_{k} \cap E_{j}=\emptyset$, if $k \neq j$. A complex measure $\lambda$ on $\mathcal{M}$ is a complex-valued function on $\mathcal{M}$ such that

$$
\lambda(E)=\sum_{k=1}^{\infty} \lambda\left(E_{k}\right)<+\infty .
$$

The total variation $|\lambda|$ of $\lambda$ is a real positive measure defined as

$$
|\lambda|(E)=\sup _{\left\{E_{k}\right\}_{k=1}^{\infty}} \sum_{k=1}^{\infty}\left|\lambda\left(E_{k}\right)\right| \quad \text { for all } E \in \mathcal{M}
$$

where $\left\{E_{k}\right\}_{k=1}^{\infty}$ is a generic partition of $E$.

The following theorem is the Lebesgue-Radon-Nikodym Theorem presented in [15, Th. $6.10]$.

Theorem 4.12. Let $\mu$ be a positive $\sigma$-finite measure on a $\sigma$ algebra $\mathcal{M}$ in a set $X$, and let $\lambda$ be a complex measure on $\mathcal{M}$. 
(a) There is then a unique pair of complex measures $\lambda_{a}$ and $\lambda_{s}$ on $\mathcal{M}$ such that

$$
\lambda=\lambda_{a}+\lambda_{s} \quad \lambda_{a} \ll \mu \quad \lambda_{s} \perp \mu .
$$

(b) There is a unique $h \in L^{1}(\mu)$, called the Radon-Nikodym derivative w.r.t. $\mu$, such that

$$
\lambda_{a}(E)=\int_{E} h d \mu .
$$

The choice $\mu=|\lambda|$ gives the following theorem [15, th. 6.12].

Theorem 4.13. Let $\lambda$ be a complex measure on a $\sigma$-algebra $\mathcal{M}$ in $X$. Then there is a function $h \in L^{1}(|\lambda|)$, called the Radon-Nikodym derivative w.r.t. $|\lambda|$, such that $|h(x)|=1$ for all $x \in X$ and such that $\lambda(E)=\int_{E} h d|\lambda|$ or, equivalently, that $d \lambda=h d|\lambda|$.

From Theorem 4.13 it follows that $\lambda(X)=\int_{X} h d|\lambda|$; furthermore, it is possible to define

$$
\int_{X} f d \lambda \stackrel{\text { def }}{=} \int_{X} f h d|\lambda|
$$

We consider a special case. Let $\mu$ be a positive real measure on $\mathcal{M}$ and let $g: X \rightarrow \mathbb{C}$ be a function in $L^{1}(\mu)$. We can define a complex measure $\lambda$ on $\mathcal{M}$ in the set $X$ as follows:

$$
\lambda(E)=\int_{E} g d \mu \quad \text { for all } E \in \mathcal{M} .
$$

Since in this case $d \lambda=g d \mu$, from Theorem 4.13 we have $g d \mu=d \lambda=h d|\lambda|$, with $|h|=1$, and so $\bar{h} g d \mu=\bar{h} h d|\lambda|=d|\lambda|$. We conclude that, in this case,

$$
\int_{X} f d \lambda=\int_{X} f h d|\lambda|=\int_{X} f h \bar{h} g d \mu=\int_{X} f g|h|^{2} d \mu=\int_{X} f g d \mu .
$$

\section{References}

[1] David R. Brillinger. Time Series, Data Analysis and Theory. Classics in Applied Mathematics. SIAM, 2001.

[2] Bruno Buchberger and H. Michael Möller. The construction of multivariate polynomials with preassigned zeros. In EUROCAM '82: Proc. of the European Computer Algebra Conf., Lect. Notes Comput. Sci., pages 24-31, 1982.

[3] Claudia Fassino, Giovanni Pistone, and Eva Riccomagno. The algebra of interpolatory cubature formulae for generic nodes. Statistics and Computing, 24:615-632, 2014.

[4] Claudia Fassino, Giovanni Pistone, Eva Riccomagno, and Maria-Piera Rogantin. Moments of the multivariate Gaussian complex random variable. arXiv:1708.09622v1 [math.PR]. 
[5] Claudia Fassino and Eva Riccomagno. The precision space of interpolatory cubature formulæ. J. Algebraic Statistics, 6(1):46-61, 2015.

[6] Roberto Fontana, Fabio Rapallo, and Maria-Piera Rogantin. Markov bases for sudoku grids. In J.M. Angulo Ibanez A. Di Ciaccio, M. Coli, editor, Advanced Statistical Methods for the Analysis of Large Data-Sets, Studies in Theoretical and Applied Statistics, pages 305-315. Springer, Berlin, 2012.

[7] Walter Gautschi. Orthogonal Polynomials - Computation and Approximation. Numerical Mathematics and Scientific Computation. Oxford University Press, New York, 2004 .

[8] Nathaniel R. Goodman. Statistical analysis based on a certain multivariate complex Gaussian distribution (an introduction). Ann. Math. Statist., 34(1):152-177, 1963.

[9] Leigh J. Halliwell. Complex random variables. Casualty Actuarial Society E-Forum, Fall 2015.

[10] Oliver Lang and Mario Huemer. Classical widely linear estimation of real valued parameter vectors in complex valued environments. arXiv:1704.08825 [math.ST].

[11] Amos Lapidoth. A Foundation in Digital Communication. Cambridge University Press, 2009.

[12] Madan M. Panja and Birendranath N. Mandal. Gauss-type quadrature rule with complex nodes and weights for integrals involving daubechies scale functions and wavelets. Journal of Computational and Applied Mathematics, 290:609-632, 2015.

[13] Giovanni Pistone, Eva Riccomagno, and Henry P. Wynn. Algebraic statistics: Computational commutative algebra in statistics. Chapman \& Hall Ltd, Boca Raton, 2001.

[14] Giovanni Pistone and Maria-Piera Rogantin. Indicator function and complex coding for mixed fractional factorial designs. J. Statist. Plann. Inference, 138(1):149-172, 2008.

[15] Walter Rudin. Real and Complex Analysis, Third Edition. Mathematics Series. McGraw-Hill International Editions, third edition, 1987.

[16] Paul E. Saylor and Dennis C. Smolarski. Why Gaussian quadrature in the complex plane? Numerical Algorithms, 26:251-280, 2001.

[17] Jiang Zhu, Rick S. Blum, and Xiaokang Lin. Robust transmit beamforming for parameter estimation using distributed sensors. IEEE Communications Letters, 20(7):1329$1332,2016$. 